\title{
Dichter dran! Neue Möglichkeiten der Vernetzung von Geobasis-, Statistik- und Erdbeobachtungsdaten zur räumlichen Analyse und Visualisierung von Stadtstrukturen mit Dichteoberflächen und -profilen
}

\author{
Stefan Fina · Angelika Krehl · Stefan Siedentop • \\ Hannes Taubenböck • Michael Wurm \\ Eingegangen: 31. Juli 2013 / Angenommen: 11. Februar 2014 / Online publiziert: 28. März 2014 \\ (C) Springer-Verlag Berlin Heidelberg 2014
}

\begin{abstract}
Zusammenfassung In der Stadtforschung und Stadtplanung fungiert die „Dichte“ als eines der wichtigsten Maße für die Beschreibung der baulichen Physis einer Stadt. Im Vergleich zu anderen Maßen - genannt seien beispielhaft die „Nutzungsmischung“, die „Polyzentralität“ oder die „Kompaktheit" - erscheint die „Dichte“ auf den ersten Blick als objektiv ermittelbar und gut verständlich. Bei näherer Betrachtung handelt es sich jedoch um ein Konzept, das sich einer einfachen empirischen Ermittlung weitgehend entzieht. Bis heute gibt es kein international anerkanntes Dichtemaß und Dichteangaben für verschiedene Länder, Regionen und Städte sind in der Regel nicht oder nur eingeschränkt vergleichbar. In diesem Beitrag werden die analytischen Möglichkeiten neuer Geodatenan-
\end{abstract}

Dr. S. Fina $(\square)$

Institut für Raumordnung und Entwicklungsplanung, Universität Stuttgart,

Pfaffenwaldring 7, 70569 Stuttgart, Deutschland

E-Mail: stefan.fina@ireus.uni-stuttgart.de

Dipl.-Volksw. A. Krehl · Prof. Dr.-Ing. S. Siedentop Institut für Landes- und Stadtentwicklungsforschung (ILS), Brüderweg 22-24, 44135 Dortmund, Deutschland

E-Mail: angelika.krehl@ils-forschung.de

Prof. Dr.-Ing. S. Siedentop

E-Mail: stefan.siedentop@ils-forschung.de

Dr. H. Taubenböck · Dr. M. Wurm

Deutsches Zentrum für Luft- und Raumfahrt (DLR),

Deutsches Fernerkundungsdatenzentraum (DFD),

82234 Weßling, Deutschland

E-Mail: hannes.taubenboeck@dlr.de

Dr. M. Wurm

E-Mail: michael.wurm@dlr.de wendungen für eine objektive und transparente Ermittlung der baulichen und nutzungsbezogenen Dichte städtischer Siedlungsgebiete aufgezeigt. Eine Fallstudie für das Gebiet der Stadt Köln demonstriert an ausgewählten Themen die Einsatzmöglichkeiten kleinräumiger Dichtedaten.

Schlüsselwörter Dichte $\cdot$ Bauliche Dichte

Nutzungsdichte $\cdot$ Monitoring $\cdot$ Fernerkundung $\cdot$ Köln

Getting Closer! New Ways of Integrating Geodata, Statistics and Remote Sensing to Analyze and Visualize Urban Structures Using Density Surfaces and -Profiles

Abstract In urban research and planning, "density" is one of the most important measures to analyze a city's structural morphology. Compared to other measures such as "neighborhood mix of uses", "polycentricity" or "compactness", density seems to be a rather objective and comprehensible choice. However, when having a closer look at it, it is a rather complex concept with different measurement options. There is no internationally accepted standard for the implementation of density or density measures that would allow for a robust comparison of different countries, regions or cities. This article deals with the analytical opportunities that new geodata options offer with regards to an objective and transparent measurement of cities' structural and use-related densities. A case study for the city of Cologne demonstrates possible applications based on highresolution and disaggregated density data.

Keywords Density · Built-up density - Urban density · Monitoring $\cdot$ Remote sensing $\cdot$ Cologne 


\section{Einführung}

In der Stadtforschung und Stadtplanung fungiert die „Dichte“ als eines der wichtigsten Maße für die Beschreibung der baulichen Physis einer Stadt, ihrer Form und ihrer Struktur (vgl. Acioly Jr./Davidson 1996; Churchman 1999; Roberts 2007; OECD 2012). Dichteindikatoren kommt sowohl als deskriptive als auch als normative Größe Bedeutung zu. Die physische Dichte anthropogener Aktivität wie des Wohnens und Arbeitens gilt als Schlüsselfaktor bei der Erklärung von standörtlichen Unterschieden des Verkehrsverhaltens (Newman/Kenworthy 1989; Forsyth/Oates/Schmitz et al. 2007; Ewing/Bartholomew/Winkelman et al. 2007; Ewing/ Cervero 2010), der Gesundheit der Bevölkerung (KellySchwartz/Stockard/Doyle et al. 2004; Hinde/Dixon 2005), der Kosten für die Bereitstellung infrastruktureller Leistungen (Doubek/Zanetti 1999; Burchell/Mukherji 2003; Siedentop/Schiller/Gutsche et al. 2006), der ökonomischen Prosperität und Innovationsfähigkeit (Bettencourt/West 2010; OECD 2012) und der mikroklimatischen Bedingungen einer Stadt (Watkins/Palmer/Kolokotroni 2007; Kropp/ Holsten/Lissner et al. 2009).

Die empirische Forschung konnte zeigen, dass mit zunehmender baulicher Verdichtung einer Stadt der Anteil der zu Fuß, mit dem Fahrrad oder mit öffentlichen Verkehrsmitteln zurückgelegten Wege steigt, da Wegelängen geringer ausfallen und die Bedienungsqualität des öffentlichen Verkehrs immer besser wird (OECD 2010 mit zahlreichen weiteren Nachweisen; OECD 2012). Das höhere Maß an nicht-motorisierter Mobilität in verdichteten Stadtgebieten verringert die Emissionen von Luftschadstoffen und Klimagasen und hat nachweislich positive Auswirkungen auf gesundheitliche Parameter der Bevölkerung (Frank/Engelke 2001). Höhere Dichten steigern ferner die Effizienz technischer Infrastrukturen, weil die Kapitalaufwendungen für die Vorhaltung von Ver- und Entsorgungsleistungen auf eine höhere Zahl von Nutzern umgelegt werden können (vgl. z. B. Siedentop/ Schiller/Gutsche et al. 2006: 6 ff. mit weiteren Nachweisen). Neuere Forschungsergebnisse zeigen darüber hinaus, dass der Grad der Verdichtung auch positive ökonomische Effekte nach sich zieht. So konnten Bettencourt und West (2010) zeigen, dass die Größe und Dichte von Städten eine exzellente Schätzgröße für die ökonomische Produktivität (Bruttoinlandsprodukt je Einwohner), das Einkommensniveau oder die Anzahl der Patente je Einwohner darstellt.

Hohe Dichten haben indes auch Nachteile, da sie mit höheren lufthygienischen sowie mikroklimatischen Belastungen einhergehen können. So ist die Vulnerabilität verdichteter Stadtbereiche in Bezug auf sommerliche Hitzestressphasen und die mit diesen verbundenen gesundheitlichen Risiken signifikant höher als in vorstädtischen oder ländlichen Siedlungsgebieten (Koppe/Kovats/Jendritzky et al. 2004). Hoch verdichtete Bereiche können ferner von
Defiziten der Freiraumversorgung betroffen sein. Schließlich wurde mit der sogenannten „Crowding-Forschung“ die Relevanz der Dichte als sozialpsychologischer Stressfaktor intensiv untersucht. Bis heute herrscht über die Wirkungen hoher Dichten auf die menschliche Psyche allerdings kein Konsens (vgl. ausführlich Newman/Hogan 1981; Churchman 1999).

Im Vergleich zu anderen Maßen der baulichen Form von Städten - genannt seien beispielhaft die „Nutzungsmischung“, die „Polyzentralität“ oder die „Kompaktheit“ erscheint die „Dichte“ auf den ersten Blick als eine einfache, objektive, gut verständliche und leicht handhabbare Größe. Bei näherer Betrachtung handelt es sich jedoch um ein Konzept, das sich einer einfachen empirischen Auseinandersetzung weitgehend entzieht. So wies Churchman Ende der 1990er Jahre (Churchman 1999: 390) darauf hin, dass es bis zum damaligen Zeitpunkt kein international anerkanntes Dichtemaß gab und dass Dichteangaben für verschiedene Länder, Regionen und Städte in der Regel nicht oder nur eingeschränkt vergleichbar sind, wenn sie aus verschiedenartigen Quellen stammen. Diese Feststellung kann nach wie vor Gültigkeit beanspruchen.

Unstrittig ist, dass Dichte ein Verhältnismaß von Objekten (dies können etwa Individuen, Arbeitsplätze oder Wohneinheiten sein) und einer Bezugsfläche darstellt, auf der diese Objekte verortet sind (vgl. ausführlich Westphal 2008: 38). Dichte wird mit einem Bruch aus der Menge der Objekte (im Zähler) und der Größe der Bezugsfläche (im Nenner) ausgedrückt. Eine grundsätzliche Unterscheidung kann zwischen baulichen Dichten und Nutzungsdichten vorgenommen werden. Bauliche Dichten beziehen sich auf die räumliche Verteilung von Baumassen, während Nutzungsdichten die Intensität der menschlichen Nutzung besiedelter bzw. bebauter Flächen ausdrücken. Erstere sind naturgemäß eher statischer Natur, da sich bauliche Strukturen zumindest in einem größeren räumlichen Zusammenhang nur sehr langsam verändern. Demgegenüber unterliegen Nutzungsdichten stetigen Veränderungen, da die ihnen zugrunde liegenden demographischen, ökonomischen und sozialen Prozesse äußerst dynamisch sind.

Im empirischen Umgang mit der Dichte einer Stadt oder eines Stadtraumes treten mannigfaltige Probleme auf, die zum einen konzeptioneller Natur sind, zum anderen mit Restriktionen bei der Verfügbarkeit von Grunddaten erklärt werden können. So leidet die Validität und Interpretierbarkeit von Dichteangaben häufig unter einer fehlenden oder unscharfen Definition des räumlichen Bezugs (Forsyth 2003: 3). Vielfach bleibt unklar, ob eine Dichte als Brutto- oder Nettogröße zu interpretieren ist, da nicht deutlich wird, welche Arten von Flächen (Grundstücksflächen, Erschließungsflächen, sonstige Siedlungsflächen) in die Dichteberechnung eingegangen sind. Zudem entstehen bei der Erhebung von Dichtewerten praktische Probleme, da 
die erforderlichen Grunddaten nicht immer verfügbar sind. Das betrifft vor allem die Ermittlung von baulichen Dichten auf der Ebene von Parzellen oder größeren Raumeinheiten. Aber auch Einwohner- oder Arbeitsplatzdichten lassen sich nicht immer in der gewünschten räumlichen Körnigkeit ermitteln, da die Verfügbarkeit von Strukturdaten auf der untergemeindlichen Ebene eingeschränkt ist.

Die flächenhafte Verfügbarkeit von Geodaten, mit denen nicht nur die Bodennutzung, sondern auch die Höhe baulicher Anlagen modelliert werden kann, bietet vor diesem Hintergrund vollkommen neue Möglichkeiten, diese Lücke zu schließen. Möglich werden großflächige Massenberechnungen und aus diesen abgeleitete Dichtemaße wie beispielsweise die Geschossflächenzahl. Vorteile ergeben sich durch

- die Objektivierbarkeit der Dichteberechnung, da sich Gebäudehöhen und -volumen eindeutig ermitteln lassen,

- die hohe räumliche Auflösung der Daten und die Möglichkeit ihrer Aggregation in beliebigen Raumbezugssystemen (wie Baublöcke, statistische Bezirke oder Raster),

- die großflächige Verfügbarkeit $\mathrm{zu}$ vergleichsweise moderaten Kosten und

- die einfache Verknüpfbarkeit mit demographischen und sozioökonomischen Strukturdaten, sofern diese auf untergemeindlicher Ebene vorliegen.

Hinzu kommt, dass auch demographische und ökonomische Strukturdaten verstärkt kleinräumig verfügbar sind. So hat beispielsweise die jüngst erfolgte Georeferenzierung der Beschäftigungsstatistik durch das Forschungsdatenzentrum der Bundesagentur für Arbeit im Institut für Arbeitsmarkt- und Berufsforschung (IAB) neue Möglichkeiten für die Untersuchung untergemeindlicher Verteilungsmuster der Arbeitsplätze eröffnet (vgl. Scholz/Rauscher/Reiher et al. 2012: 4 ff.). Ähnliches kann für die Bevölkerungsstatistik ausgesagt werden, da auch hier neue Datenangebote existieren. Verwiesen sei auf die Innerstädtische Raumbeobachtung des Bundesinstituts für Bau-, Stadt- und Raumforschung (BBSR) oder den KOSTAT-Datensatz der kommunalen Statistikämter. ${ }^{1}$

In diesem Beitrag sollen die analytischen Möglichkeiten neuer Geodatenanwendungen für die Ermittlung der baulichen und nutzungsbezogenen Dichte städtischer Siedlungsgebiete aufgezeigt werden. Nach einer knappen Darstellung des wissenschaftlichen Hintergrunds (Kap. 2) wird die methodische Vorgehensweise bei der Erzeugung kleinräumig differenzierter Dichtedaten vorgestellt. Ausführlich wird dabei auf die Erhebung von geometrisch detaillierten räumlichen Grundlagendaten mit Methoden der Erdbeobachtung eingegangen (Kap. 3). Anhand einer kommunalen Fallstudie (Stadt Köln) soll in einem weiteren Schritt das

\footnotetext{
${ }^{1}$ Vgl. http://www.staedtestatistik.de/kostat.html (17.01.2014)
}

analytische Potenzial der auf diese Weise erzeugten Informationen für die Stadtforschung und -planung aufgezeigt werden (Kap. 4). Ziel ist es letztendlich, ein umfassendes Bild der Morphologie einer Stadt zu erzeugen und die methodisch-analytischen Möglichkeiten diesbezüglicher Daten in verschiedenen Anwendungsbereichen der Stadtund Raumforschung sowie der Planungspraxis aufzuzeigen. Abschließend erfolgt ein knappes Fazit mitsamt einer Diskussion perspektivischer Anwendungsmöglichkeiten des Datenbestands (Kap. 5).

\section{Wissenschaftlicher Hintergrund}

Die Diskussion um die ,richtige " oder die ,angemessene Dichte einer Stadt und ihrer Teilräume ist vielleicht so alt wie die Disziplin der Stadtplanung selbst und sie kann zweifelsohne als eine der kontroversesten gelten (Gassner 1994; Westphal 2008). „Dichte“ ist ein ausgeprägt interdisziplinäres Konzept (vgl. ausführlich Roskamm 2011), dessen Bedeutungsgehalt im Zeitverlauf immer wieder Veränderungen ausgesetzt war. Unstrittig ist, dass die Wahrnehmung und Bewertung von Dichte in hohem Maße kulturellen und sozialen Prägungen unterliegt (Acioly/Davidson 1996: 6). Ein objektiver Maßstab, mit dem bauliche oder nutzungsbezogene Dichten als ,hoch“, ,gering“" oder gar als ,,angemessen" zu bewerten sind, kann vor diesem Hintergrund nicht erwartet werden.

Der Umgang mit dem „Konzept Dichte“ ist aber nicht nur aus normativ-planerischer Perspektive problembehaftet. Auch eine objektive Messung der städtischen Dichte ist verschiedenen Restriktionen ausgesetzt, die in der Einleitung bereits angesprochen wurden. Grundsätzlich gilt, dass die Schwierigkeiten der Ermittlung von Dichtewerten positiv mit dem zugrunde gelegten Maßstab korrelieren. Insbesondere auf der Ebene von Parzellen, Blockseiten oder Baublöcken fehlt es häufig an genauen und aktuellen Dichtewerten. Der Grund hierfür ist, dass die amtliche Statistik in Deutschland im Regelfall keine Daten unterhalb der Gemeindeebene bereitstellt (bzw. bereitstellen darf). Studien, die sich auf eigene Erhebungen (z. B. durch Ortsbegehungen) oder auf private Geodatenangebote stützen, sind entweder aus Aufwandsgründen oder aus Gründen der intransparenten Validität der Daten in ihren Möglichkeiten eingeschränkt. Bis heute werden Dichtestudien meist in Form von kommunalen Einzelanalysen durchgeführt (vgl. z. B. Landeshauptstadt Stuttgart 2005), deren Ergebnisse oftmals nur begrenzt mit anderen Kommunen oder Gebietseinheiten vergleichbar sind.

Die Modellierung von urbanen Dichteoberflächen und -profilen leidet daher an einer geringen räumlichen Körnigkeit. Dies wirkt sich nicht nur negativ auf die Planungspraxis aus (z. B. im Rahmen der Modellierung von Lärmbelastungen 
oder der Durchführung von klimatischen Vulnerabilitätsstudien), sondern behindert auch die Stadt- und Raumforschung. Die Bedeutung der Dichte als Schätzgröße bei der Erklärung der räumlichen Varianz bestimmter Phänomene (wie z. B. der standortspezifischen Verkehrsmittelwahl oder der Infrastrukturkosten) stellt eigentlich hohe Anforderungen an die Qualität verfügbarer Daten. In vielen Studien müssen jedoch einfache Bevölkerungs- oder Siedlungsdichten verwendet werden, da präzisere Dichteindikatoren nicht verfügbar sind.

Besonders große Schwierigkeiten entstehen bei der Abbildung baulicher Dichten. Meistens bezieht sich dies auf das Verhältnis von Gebäudegeschossfläche oder -volumen zur Grundfläche. Ein Standardindikator ist dabei die Geschossflächenzahl bzw. -dichte (GFZ bzw. GFD, im Englischen als Floor Area Ratio, Floor Space Ratio oder Floor Space Index bezeichnet). Die Ermittlung der Geschossflächendichte ist voraussetzungsvoll, weil in der Regel keine amtlichen Erhebungen zu Gebäudemaßen (z. B. Volumenoder Höhenangaben) verfügbar sind. Zwar existieren viele Studien, die auf der Ebene von ausgewählten Baugebieten oder Strukturtypen Spannbreiten der baulichen Dichte (z. B. die GFZ) angeben. Häufig sind deren Ergebnisse aber nicht vergleichbar, da unterschiedliche Definitionen von Dichte verwendet werden oder sich die Vorgehensweise bei der Erhebung unterscheidet.

Dies lenkt den Blick auf ein zweites, eher konzeptionelles Problem: Der Umgang mit Dichteindikatoren leidet häufig unter einer nicht präzisen Definition der Bezugsfläche. So ist zum Teil nicht immer deutlich, ob die Gesamtfläche einer Gebietseinheit oder nur die von einer bestimmten Nutzung belegte Fläche (z. B. Wohnbaufläche) in die Berechnung eingeht. In derartigen Fällen bleibt es unklar, ob sich die Angabe als Brutto- oder Nettodichte interpretieren lässt. Bei einer Nettodichte werden die gezählten Objekte auf die relevante Nutzfläche bezogen (z. B. die Anzahl der Einwohner auf Wohnbauflächen). Sonstige Bodenflächen wie Verkehrserschließungsflächen, Grün- und Erholungsflächen, gewerbliche Nutzungen oder gar land- und forstwirtschaftlich genutzte Flächen bleiben unberücksichtigt. Nettodichten informieren in weitaus geeigneterer Form über die „Realität“ der Verdichtung, da sie weniger stark vom räumlichen Zuschnitt des Untersuchungsraumes abhängig sind. Das sogenannte Modifiable Areal Unit Problem (MAUP), wonach die Aggregation von Messergebnissen zu räumlichen Phänomenen (z. B. Dichtewerte) mit der Größe des zugrunde gelegten Untersuchungsgebiets variiert, kann durch die Verwendung von Nettodichten begrenzt werden (vgl. Abb. 1; vgl. auch Forsyth 2003). Ein Großteil der in der Literatur anzutreffenden Dichteangaben sind allerdings Bruttodichten (vgl. z. B. Senatsverwaltung für Stadtentwicklung und Umwelt Berlin 2013).

Im weiteren Teil dieses Beitrags werden methodische Möglichkeiten aufgezeigt, um mit neuartigen Daten der

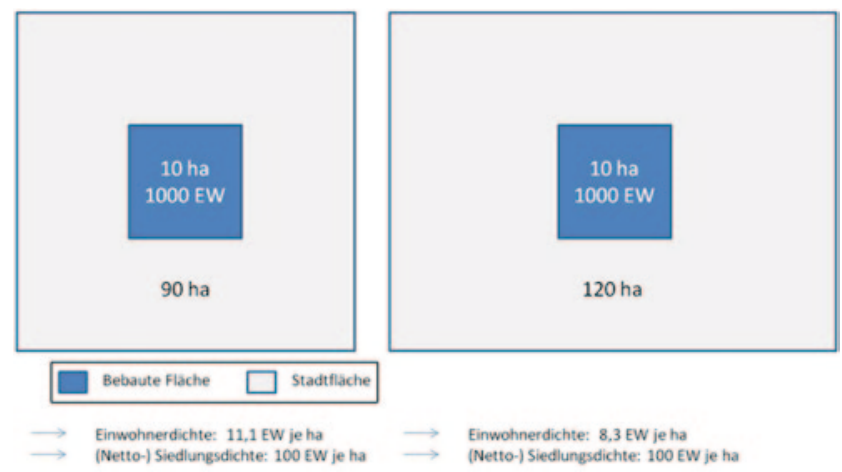

Abb. 1 Modifiable Areal Unit Problem: Die Einwohnerdichte eines Gebiets verringert sich, wenn sich die administrative Bezugsfläche (grau) verändert; die Siedlungsdichte bleibt hingegen konstant, da sich die besiedelte Fläche (blau) nicht verändert hat

Fernerkundung sowie der Bevölkerungs- und Beschäftigungsstatistik Dichteeigenschaften von Städten hochauflösend zu erfassen.

\section{Verfahren zur Berechnung der physischen Dichtekennwerte}

In diesem Kapitel wird das Verfahren zur Ermittlung baulicher Dichtemaße dargestellt. Eingesetzt werden Daten für das Gebiet der Stadt Köln. Die Ergebnisse werden im nachfolgenden Kap. 4 hinsichtlich ihrer planungs- und forschungspraktischen Relevanz mit Daten zu kleinräumigen Einwohner- und Arbeitsplatzdichten zusammengeführt.

Die Beschreibung und Quantifizierung von baulichen Dichteparametern beruht auf der Aggregation von individuellen Objektparametern zu einer übergeordneten räumlichen Ebene. Im konkreten Anwendungsfall der Bebauungsdichten ist damit die Beziehung zwischen physiognomischen Gebäudemerkmalen und Baublöcken gemeint. Die Erzeugung der individuellen Gebäudemerkmale wird durch neue Technologien in der Geodatenerfassung erheblich erleichtert, da nicht mehr ausschließlich auf Daten der amtlichen Landes- und kommunalen Vermessungsämter zurückgegriffen werden muss oder Vor-Ort-Erhebungen durchzuführen sind. Das Verfahren zur Herstellung der räumlichen Grundlagendaten und Berechnung der physischen Dichtemerkmale wird im Folgenden beschrieben.

\subsection{Flugzeuggetragenes Laserscanning}

Mit Aufnahme- und Auswertungsverfahren der Erdbeobachtung können flächendeckende, aktuelle Daten der physischen Struktur der Erdoberfläche und, seit dem Aufkommen geometrisch besonders hoch aufgelöster Bilddaten, insbesondere die städtische Struktur erfasst werden. Dabei stellt die Datenaufnahme durch flugzeuggetragenes Laserscan- 
ning ein etabliertes Verfahren für die Herstellung von digitalen Oberflächenmodellen dar, das bereits seit vielen Jahren nicht nur im wissenschaftlichen Kontext zur Anwendung kommt (Ackermann 1999). Diese Daten sind die Grundlage für die Ableitung von physiognomischen Merkmalen der Einzelgebäude. Bei dieser digitalen Akquisition von Informationen der Erdoberfläche wird in der Regel ein Flugzeug mit einem Laserscanner über dem Beobachtungsgebiet zum Einsatz gebracht. Der Scanner sendet dabei Laserpulse aus, welche von Objekten auf der Erdoberfläche reflektiert werden und wieder vom Scanner erfasst werden. Über die Messung der Laufzeit der einzelnen Laserpulse und die absolute äußere und innere Orientierung des Scanners mittels Inertialem Navigationssystem (INS) ${ }^{2}$ und Globalem Positionierungssystem (GPS) kann die exakte Distanz zum Scanner und daraus folgend die exakte räumliche Position des Objektes auf der Erdoberfläche erfasst werden (vgl. z. B. Albertz 2001; Lillesand/Kiefer/Chipman 2004). Bei den reflektierten Laserpulsen werden dabei first pulse, last pulse und full wave form pulse unterschieden. Die Bezeichnungen richten sich nach der Reihenfolge der Reflexion, wonach zuerst die Pulse von den höchsten (in der Regel gehört die Vegetation bzw. Gebäude auf der Erdoberfläche dazu) Objekten reflektiert werden und dann von den niedrigsten Objekten, zumeist die Geländeoberfläche. Die letzte Form der Datenspeicherung beinhaltet sämtliche Informationen.

Aus der gewonnenen, diskreten Punktwolke der Einzelmessungen werden zu Darstellungs- und Analysezwecken kontinuierliche Oberflächen mittels Interpolationsverfahren berechnet. Diese, die Geländehöhen inklusive der Objekthöhen beinhaltenden Modelle werden als Digitale Oberflächenmodelle (DOM) bezeichnet. Für die Verarbeitung dieser dreidimensionalen Daten im urbanen Anwendungsfeld hat sich die Auswertung von Digitalen Oberflächenmodellen aus last pulse Daten etabliert, da diese Daten hauptsächlich physische Bebauungsstrukturen bzw. die Geländeoberflächen enthalten und die Vegetation in diesen Daten weitgehend nicht enthalten ist. Höhenmodelle, welche nur die Höheninformationen des Geländes ohne die darauf befindlichen Objekte beinhalten, werden als Digitale Geländemodelle (DGM) bezeichnet. Sie werden zumeist über Filterungsverfahren direkt aus dem Digitalen Oberflächenmodell oder aus anderen Quellen (z. B. Isohypsen aus topographischen Karten) hergestellt.

Für das Untersuchungsgebiet der Stadt Köln wurde sowohl ein digitales Oberflächenmodell aus last pulseDaten aus einer flugzeuggetragenen Laserscannerbefliegung vom 4. Oktober 2007 verwendet als auch ein aus derselben

\footnotetext{
${ }^{2}$ Mit dem „Trägheitsnavigationssystem“ (Inertialem Navigationssystem (INS)) werden über Sensoren die Bewegungen von im Raum frei beweglichen Körpern gemessen.
}

Quelle stammendes Digitales Geländemodell. Die Gitterweite der Daten beträgt $1 \times 1 \mathrm{~m}$.

\subsection{Extraktion von Einzelgebäuden aus dem Digitalen Oberflächenmodell}

Das Digitale Oberflächenmodell bildet die Grundlage für die Herstellung der räumlichen Basisinformation zur Ableitung der städtischen morphologischen Dichtemassen. Da die darin enthaltenen Höheninformationen neben den Objekthöhen aber auch Geländehöhen beinhalten, ist vor der Auswertung der Höheninformationen eine Normalisierung durchzuführen. Sie stellt die Reduktion der Höhenwerte um die Höhen des Geländes dar. Dafür werden die Höhenwerte des Digitalen Geländemodells von den Höhenwerten des Digitalen Oberflächenmodells subtrahiert. Als Ergebnis liegt nach diesem Verarbeitungsschritt ein normalisiertes digitales Oberflächenmodell (nDOM) vor. Eine Gegenüberstellung der Datensätze für die Normalisierung ist in Abb. 2 dargestellt.

Aus dem normalisierten Oberflächenmodell können im folgenden Verarbeitungsschritt Daten auf der räumlichen Ebene der Einzelgebäude gewonnen werden. Dafür müssen zunächst die aneinandergereihten, aber zusammenhanglosen Bildpunkte des normalisierten Oberflächenmodells zu semantischen Bildpunktverbänden zusammengefasst werden. Sie repräsentieren Gebäudegrundflächen, für welche die relative Höhe bekannt ist. Die Ableitung dieser Gebäudegrundflächen geschieht basierend auf der Hypothese, dass Gebäude erhabene Objekte auf der Erdoberfläche darstellen und entsprechend eine größere Höhe als ihre Umgebung aufweisen. Im normalisierten Oberflächenmodell sind diese relativen Höhen als hellere Bildpunkte zu erkennen (vgl. Abb. 2). Dieser Grauwertunterschied im Bild wird für die Abgrenzung der Einzelgebäude in der computergestützten Bildanalyse genutzt. Das Bildanalyseverfahren untersucht den lokalen Grauwertunterschied des Bildes und ermittelt in einem empirischen Verfahren den besten Kontrast. Dafür werden für die einzelnen Baublöcke aus dem Basis-Digitalen Landschaftsmodell (Basis DLM) des amtlichen topographisch-kartographischen Informationssystems (ATKIS) die Kontraste ermittelt. Die Berechnung des höchsten Kontrastes ist ein iteratives Verfahren, welches wiederholt den Kontrast zwischen zwei Testgrauwerten auswertet. Diese Testschwellwerte liegen zwischen dem lokal kleinsten und dem lokal größten Grauwert. Zwischen den Grauwertbereichen, für welche der höchste Kontrast gefunden wurde, wird ein Schwellwert gesetzt, welcher die erhabenen (helleren) Objekte von den niedrigen (dunklen) Objekten trennt. Dieser Schritt wird für jeden Baublock durchgeführt. Für die Berechnung des Kontrastes wurde im verwendeten Verfahren die Kantenratio verwendet. Sie stellt eine Weiterentwicklung der Auswertung des Kontrastes auf Basis der 


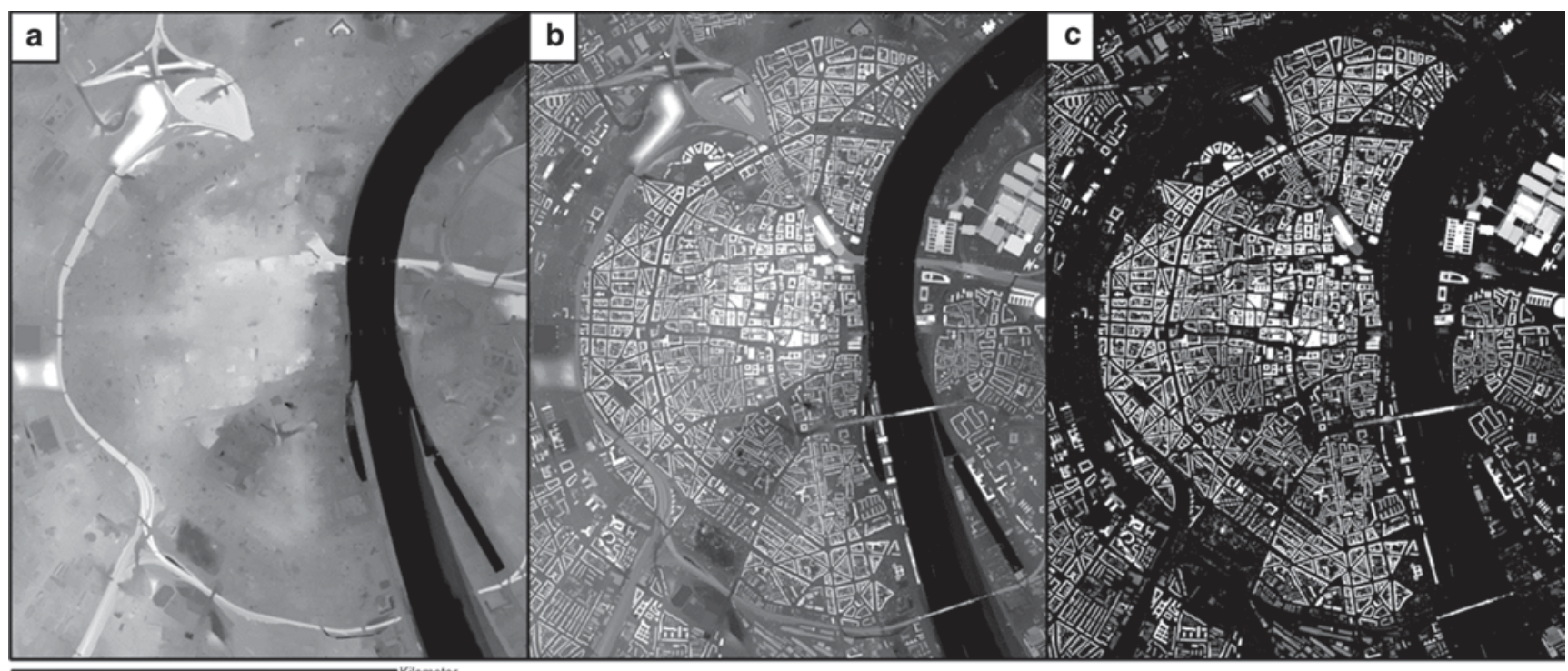

Abb. 2 Gegenüberstellung der unterschiedlichen Höheninformationen. a DGM, b DOM, und c nDOM

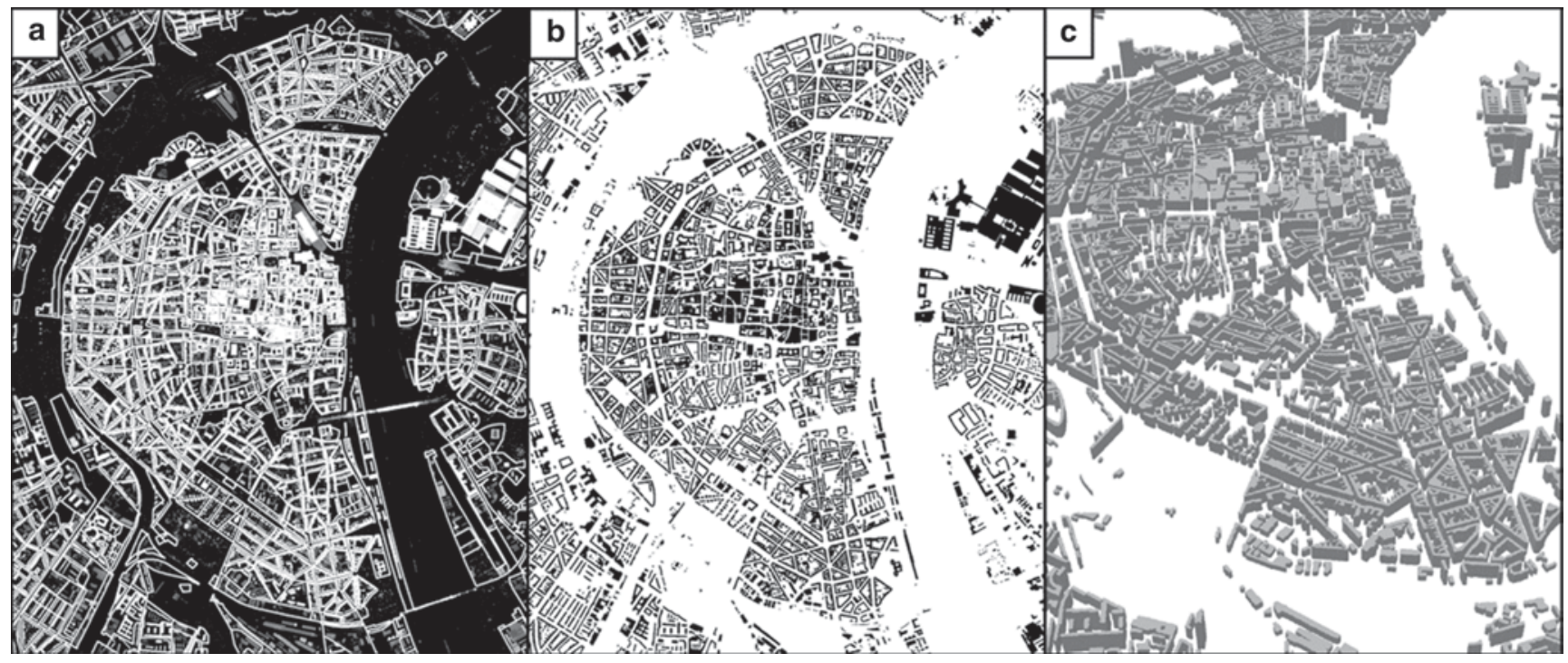

Abb. 3 Ableitung des Gebäudemodells aus der Höheninformation a Überlagerung des nDOMs mit den ATKISBaublöcken, b abgeleitete Einzelgebäude, und c 3D-Visualisierung

Objektdifferenzen dar. Bei der Kantenratio wird der Kontrast zwischen den Testgrauwerten der potenziellen Kanten, die helle von dunklen Objekten unterscheiden, ausgegeben. Sie wird auf der Basis folgender Gleichung berechnet:

$$
k=\frac{a-b}{a+b} \text { mit } a \geq b \geq 0
$$

wobei $k$ der Kontrast ist, $a$ der Mittelwert der Grauwerte der hellen Kantenobjekte und $b$ der Mittelwert der Grauwerte der dunklen Objekte. Die Grauwerte der erhabenen Objekte im normalisierten Oberflächenmodell sind immer größer oder gleich Null, weshalb der Kontrast immer positiv ist.
Die Grauwerte innerhalb jedes ATKIS-Baublockes werden nach der Ermittlung des höchsten Kontrastes in helle - also erhabene - und dunkle - also niedrige - Bereiche unterteilt. Die erhabenen Bereiche repräsentieren entsprechend auch die Gebäude, welche aus zusammenhängenden Bildpunktverbänden bestehen. Somit ist jedes Gebäude als eigenständiges Objekt definiert und durch eine Gebäudegrundfläche repräsentiert. Diese individuellen Gebäudegrundflächen, welche zunächst als zweidimensionale, flächenhafte Information vorliegen, werden mit den relativen Höheninformationen aus dem normalisierten Oberflächenmodell kombiniert und resultieren in einem dreidimensionalen Gebäudemodell. In Abb. 3 sind die oben beschriebenen 
Verfahrensabläufe chronologisch visualisiert. Sie zeigen das normalisierte Oberflächenmodell mit den überlagerten Baublöcken, für das die lokal höchsten Kontraste berechnet wurden, die abgeleiteten Gebäudegrundflächen und eine perspektivische Darstellung des dreidimensionalen Gebäudemodells. Detaillierte Informationen zur Berechnung der Kontraste können bei Wurm (2013: 72 ff.) nachgelesen werden.

Neben der Gebäudegrundfläche und der metrischen Höhe der Einzelgebäude ist für die Berechnung von Dichtekennwerten auch die Geschossigkeit der Gebäude von Relevanz. Sie ist nicht bekannt und kann auch nicht direkt aus den Höhendaten ermittelt werden. Allerdings kann über eine einfache Hypothese ein Zusammenhang zwischen der Geschossigkeit und der bekannten metrischen Gebäudehöhe hergestellt werden. Die Hypothese orientiert sich an der Feststellung, wonach in der Regel Gebäude mit einer hohen Geschossigkeit auch eine hohe metrische Höhe aufweisen und je größer die metrische Höhe ist, desto größer ist in der Regel auch die Geschossigkeit. Für die Überprüfung und Quantifizierung dieses Zusammenhangs wird ein empirisches Modell anhand bekannter Geschossigkeiten und Gebäudehöhen erstellt. Dafür werden exakte Gebäudehöheninformationen aus offiziellen Referenzdaten des amtlichen Gebäudekatasters verwendet (vgl. Wurm/Taubenböck/ Schardt et al. 2011). Der ermittelte empirische Zusammenhang zwischen der metrischen Gebäudehöhe und der Geschossigkeit ist in Gl. (2) festgehalten.

$$
h=5,59 * g^{0,73}
$$

wobei $h$ die metrische Gebäudehöhe darstellt und $g$ die Geschossigkeit. Aus dieser Gleichung kann für jedes Gebäude, dessen metrische Höhe bekannt ist, die Geschossigkeit abgeschätzt werden. Die ermittelte Beziehung der Geschossigkeit mit der Gebäudehöhe auf Basis der Formel stellt eine nichtlineare Potenzregression dar, welche bereits unterschiedliche Geschosshöhen von Gebäuden unterschiedlicher Epochen zumindest ansatzweise berücksichtigt. Eine einheitliche Höhe für eine lineare Beziehung führt aufgrund der stark unterschiedlichen Geschosshöhen zu Überschätzungen der Geschossigkeiten für typische Altbauten mit Raumhöhen bis zu 4 m und zu Unterschätzungen für Hochhausbauten mit mittleren Geschosshöhen von etwa 2,2 m. Eine Visualisierung des Zusammenhangs findet sich in Abb. 4. Im Vergleich der inneren statistischen Annahme mit den Kalibrierungsdaten zeigt sich, dass für knapp $60 \%$ aller Gebäude die exakte Geschossigkeit abgeschätzt werden kann und mit einer Fehlertoleranz von einem Geschoss über $90 \%$ Übereinstimmung erreicht werden (Wurm/ Taubenböck/Schardt et al. 2011: 140). Die Abweichungen ergeben sich aus der mathematischen Annahme der Hypothese, dass die Geschosshöhe für sämtliche Gebäude gleich

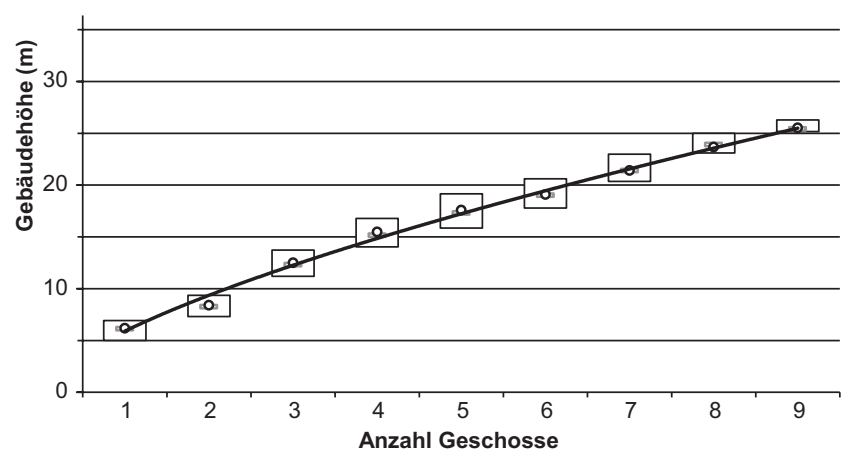

Abb. 4 Empirische Ermittlung der Geschossigkeit der Gebäude

Tab. 1 Gebäudespezifische Maßzahlen

\begin{tabular}{llllll}
\hline & Fläche & Höhe & Volumen & Geschossigkeit & Wohnraum \\
\hline Kürzel & $\mathrm{A}$ & $\mathrm{h}$ & $\mathrm{V}$ & $\mathrm{g}$ & $\mathrm{W}$ \\
Einheit & $\mathrm{m}^{2}$ & $\mathrm{~m}$ & $\mathrm{~m}^{3}$ & Anzahl Geschosse & $\mathrm{m}^{2}$ \\
\hline
\end{tabular}

groß ist und dass Ausbauten von Dachgeschossen für Wohnnutzungen aus den Höhendaten nicht ableitbar sind. Darüber hinaus wirkt sich auch die Dachform auf die ermittelte Geschossigkeit aus.

Aus den oben beschriebenen gebäudespezifischen, physiognomischen Merkmalen können weitere quantitative Maßzahlen berechnet werden (vgl. Tab. 1), welche für die Berechnung der blockspezifischen Dichtekennwerte relevant sind. Damit sind allerdings noch keine Nutzungseffizienzwerte gemeint, die einer Verknüpfung dieser Kennwerte mit der Anzahl der Nutzer bedarf (z. B. für den Wohnraum: Anzahl Einwohner pro Wohnung $=$ Wohnraumbelegung).

\subsection{Berechnung der blockspezifischen Dichtekennwerte}

Aus den im vorigen Abschnitt beschriebenen gebäudespezifischen Maßzahlen werden in der Folge die blockspezifischen Dichtekennwerte berechnet. Dafür wird jeweils die Gesamtheit der gebäudespezifischen Maßzahlen zur räumlich übergeordneten Einheit des Baublocks in Bezug gesetzt.

Bebaunngsdichte Die Bebauungsdichte definiert sich als Anteil der Gebäudefläche pro Baublock. Im Planungsund Baurecht entspricht sie der Grundflächenzahl (GRZ). Der Baublock stellt dabei die Begrenzung einer Bebauung durch das umgebende Straßengeviert dar. Die räumliche Abgrenzung ist eine gängige Raumeinheit in der Stadtplanung und wird in dieser Form durch das Bundesamt für Kartographie und Geodäsie (BKG) im Basis-DLM von ATKIS als Sachthema ausgegeben. Für die Berechnung der Bebauungsdichte werden sämtliche Gebäudegrundflächen pro Baublock kumuliert und durch die Fläche des ATKISBaublocks dividiert. Der proportionale Bezug stellt das Verhältnis der Gebäudefläche zur Baublockfläche dar und kann zwischen 0 und $100 \%$ liegen. 


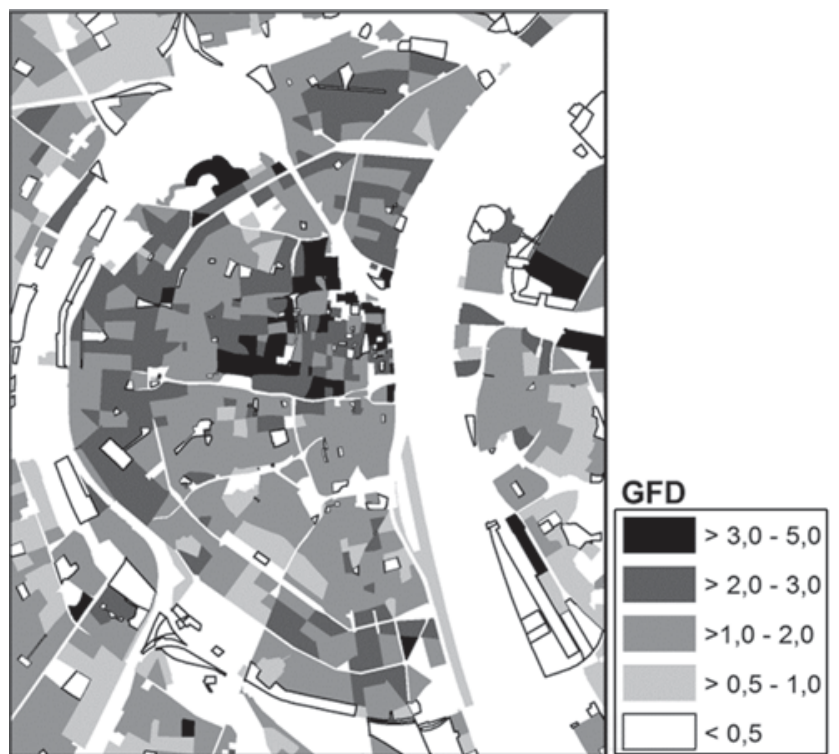

Abb. 5 Geschossflächendichte (GFD) für einen Ausschnitt der Kölner Innenstadt

Volumendichte Die Volumendichte stellt den proportionalen Volumenanteil sämtlicher Gebäude zur Fläche des Baublocks dar und berechnet sich aus der Summe der Volumina der Einzelgebäude im Verhältnis zur Grundfläche des Baublocks.

Geschossflächendichte Die Geschossflächendichte ist ein $\mathrm{Ma}$ der baulichen Dichte, welches vor allem im Städtebau zur Anwendung kommt. Sie berechnet sich analog zur Bebauungsdichte, mit dem Unterschied, dass auch die Geschossigkeiten der Gebäude berücksichtigt werden. Es wird nicht nur die Summe der Gebäudegrundfläche mit der ATKIS-Baublockfläche in Beziehung gesetzt, sondern die Summe der Flächen sämtlicher Geschosse. Die Geschossflächendichte kann daher im Gegensatz zur Bebauungsdichte ein Vielfaches der zur Verfügung stehenden Bezugsfläche des ATKIS-Baublocks sein. Ein Beispiel soll dies verdeutlichen: eine Geschoßflächendichte von 1 kann eine eingeschossige Bebauung der gesamten ATKIS-Baublockfläche bedeuten. Es ist allerdings auch möglich, dass nur die Hälfte der zur Verfügung stehenden ATKIS-Baublockfläche mit einem zweigeschossigen Gebäude bebaut ist. Die Summe der Gebäudeflächen ist in beiden Fällen die gleiche.

Für einen Ausschnitt der Kölner Innenstadt ist die Geschossflächendichte in Abb. 5 dargestellt.

\section{Einsatzmöglichkeiten von Dichtedaten in der Stadtplanung und Stadtforschung}

Räumlich hoch auflösende Messwerte zu baulichen Dichten, deren Ermittlung im vorangegangenen Abschnitt beschrieben wurde, lassen sich mit demographischen und ökonomischen Daten verknüpfen, was viele neue Anwendungsmöglichkeiten für die Stadtplanung und Stadtforschung bietet. Möglich wird erstmals ein integriertes, multidimensionales Dichtemonitoring, welches verschiedene Dichteindikatoren zusammenführt. Nachfolgend wird zunächst die Vorgehensweise bei der Errechnung von baublockscharfen Einwohner- und Arbeitsplatzdichten vorgestellt, bevor beispielhafte Anwendungen für die Stadt Köln für den aktuell verfügbaren Zeitschnitt aufgezeigt werden. Ein echtes Monitoring würde darauf aufbauend wiederholte Beobachtungen ermöglichen und Veränderungen von Dichtewerten erfassen.

\subsection{Erzeugung kleinräumiger Daten zu Bevölkerungs- und Arbeitsplatzdichten}

Für die Stadtforschung und Stadtplanung sind neben baulichen Dichten Nutzungsdichten wie die Einwohner- oder die Arbeitsplatzdichte von zentraler Bedeutung. Hierfür wurden die oben beschriebenen fernerkundlich erhobenen Baumassen mit statistischen Daten der Stadt Köln (Einwohnerzeitreihen auf Baublockebene, digitaler Flächennutzungsplan 2012), amtlichen Geobasisdaten (ATKIS Basis-DLM) und disaggregierten Beschäftigungsdaten des Instituts für Arbeitsmarkt- und Berufsforschung (IAB) (vgl. Scholz/ Rauscher/Reiher et al. 2012) kombiniert. Im Ergebnis stehen grafische Auswertungen, die Trends und Zustand von Dichteverteilungen über das Stadtgebiet veranschaulichen.

Bei der Berechnung von Einwohner- und Arbeitsplatzdichten ist zu beachten, dass aus Datenschutzgründen keine adress- oder gebäudescharfen Daten zur Verfügung gestellt werden können. Möglich ist indes die Verknüpfung mit adressscharfen Daten in einer vorgegebenen Gebietsstruktur. Mit der Baublockebene wurde in diesem Zusammenhang eine Raumbezugsebene verwendet, die ausreichend kleinräumig und gleichzeitig nur mit geringen Restriktionen durch Datenschutzbestimmungen konfrontiert ist.

Von Seiten der Stadt Köln wurden Baublöcke mit Einwohnerdaten ohne Verkehrsflächen und Innenhöfe bereitgestellt. Die daraus resultierenden Dichtewerte sind als Nettodichte anzusehen. Um zusätzlich auch Bruttodichten ermitteln zu können, wurden die Baublockdaten auf die Flächennutzungsdaten von ATKIS umgelegt. Dabei kam folgendes Verfahren zum Einsatz: Die Baublöcke der Stadt Köln wurden räumlich mit allen ATKIS-Flächen verschnitten und daraus ein Anteilsfaktor für die Einwohner in der geschnittenen Fläche berechnet. Dieser Faktor basiert auf dem Flächenanteil der geschnittenen Fläche am ursprünglichen Baublock und wird auf die Einwohnerzahlen angewandt.

Die baulich geprägten Flächen aus ATKIS (Objektarten: 2111, 2112, 2113 und 2114) stimmen überwiegend mit den Baublöcken der Stadt Köln, weshalb die dafür berechneten Einwohnerdichten als hoch plausibel anzusehen sind. Aus 
Gründen der Anwendbarkeit des beschriebenen Verfahrens wurden mögliche Umlegungen von Einwohnern aus Baublöcken auf nicht bewohnte ATKIS-Blöcke nicht weiter analysiert. Dies stellt einen Sonderfall dar und kommt nur vor, wenn durch Digitalisierungsunterschiede kleinere Baublockteile unbewohnte ATKIS-Flächen überlagern. Die flächenproportionale Umlegung stellt hier sicher, dass die Einwohnerdichte für diese Überstände entsprechend niedrig bleibt. In die Weiterverarbeitung der berechneten Daten gehen also nur ATKIS-Flächen mit plausiblen Einwohnerdichten ein.

Parallel zur Berechnung von Einwohnerdichten wurden ebenfalls auf der räumlichen Ebene der Baublöcke die Arbeitsplatzdichten ermittelt. Vom IAB werden lediglich Angaben zu sozialversicherungspflichtig Beschäftigten abgegeben, aus Datenschutzgründen nur für die von den Autoren zur Verfügung gestellten ATKIS-Flächen, die eine rechtlich unbedenkliche Anzahl an Betrieben enthalten. Für alle anderen Baublöcke können keine Informationen zur Verfügung gestellt werden (=Schwärzung, vgl. Bundesagentur für Arbeit 2012). Um auch mit ,geschwärzten“ Baublöcken arbeiten zu können, wurde hier ein Wert von exakt einem sozialversicherungspflichtig Beschäftigten angenommen. Dieser hypothetische Wert kann nur dann zu einer kritischen Verzerrung führen, wenn ein Baublock von sehr wenigen Betrieben mit hohen Beschäftigtenzahlen dominiert wird. Eine für die Datenschutzanforderungen zu kleine Anzahl an sozialversicherungspflichtig Beschäftigten wird damit adäquat repräsentiert.

Es muss weiterhin darauf hingewiesen werden, dass die vorliegenden Daten zur Beschäftigung die Konzentration tendenziell überschätzen. An mehreren Standorten innerhalb Kölns agierende Unternehmen können alle Mitarbeiter an einer Adresse im Stadtgebiet melden, wenn die Mitarbeiter in demselben Wirtschaftszweig (,5-Steller" der Wirtschaftszweigklassifikation der Bundesagentur für Arbeit von 2008) angemeldet sind. Sind die Mitarbeiter in unterschiedlichen Wirtschaftszweigen gemeldet, müssen sie an einer Stelle gemeldet werden, an der sie entweder selbst oder weitere Beschäftigte desselben Wirtschaftszweiges arbeiten.

\subsection{Erzeugung von „Heat Maps“ zu städtischen Dichteoberflächen}

Die erzeugten baublockscharfen Dichtedaten über Bewohner und Beschäftigte lassen im Folgenden differenzierte Analysen und kartographische Anwendungen zu. So können beispielsweise Techniken des Heat Mappings angewendet werden. Diese Visualisierungsmethode ermöglicht eine kartographische Herausarbeitung der generellen räumlichen Trends in den Datenpunkten. Eine thematische Karte auf Baublockebene (mit 20.624 Flächen) wäre kaum darstellbar und zu detailliert, um auf den ersten Blick Muster erkennen zu können. Die Einwohnerdichte für die ATKISBlöcke wird damit zur Grundgesamtheit für die räumliche Interpolation nach dem Verfahren des Diffusion Kernel ${ }^{3}$ (Gauß'sche Verteilung mit einer Bandbreite von $500 \mathrm{~m}$ ), die die Dichtewerte über die Stadt Köln generalisiert (vgl. Abb. 6). Für das Heat Mapping wurden bewusst keine Barrieren gesetzt, das heißt, die Interpolation verläuft frei im Raum über Verkehrsflächen und auch topographisch-geographische Barrieren wie den Rhein hinweg. Grund hierfür ist die Annahme, dass in einem hoch verdichteten Raum wie der Stadt Köln derartige Schranken kaum Einfluss auf die Verteilung der Einwohnerdichte haben. Anders formuliert: Die Attraktivität benachbarter Baublöcke ist unabhängig von einer eventuellen räumlichen Trennung durch physische oder natürliche Barrieren gegeben, sondern orientiert sich eher an anderen Aspekten wie der VerkehrserschlieBung oder dem Bodenpreis.

Zunächst werden die Ergebniskarten für die errechneten Dichtekennwerte (morphologische Dichtekarten der Stadt Köln) vorgestellt. Abbildung 6 zeigt in der oberen Reihe links die Einwohnerdichte, gemessen in Einwohner je Hektar (Stand 2011). Während die gesamte Stadt im arithmetischen Mittel eine Einwohnerdichte von 25,5 Einwohnern pro Hektar hat, liegt der Mittelwert für die Arbeitsplatzdichte bei 7,4 Beschäftigten pro Hektar (oben rechts) bzw. bei 2,5 Dienstleistungsbeschäftigten ${ }^{4}$ pro Hektar (unten rechts). Für die Baumassendichte ergibt sich arithmetisch ein Mittel von knapp $8.000 \mathrm{~m}^{3}$ pro Hektar (unten links).

Erwartungsgemäß sind die Dichten bei allen Indikatoren im (erweiterten) Innenstadtbereich am höchsten und nehmen tendenziell zum Stadtrand hin ab. Die Bevölkerung ist im Zentrum und in zentrumsnahen Wohnschwerpunkten konzentriert. Bemerkenswert ist der hohe Wohnanteil der innerstädtischen Gebiete in Köln, was auf ausgeprägte Mischstrukturen schließen lässt. Trotz einer auch in Köln in den vergangenen Jahrzehnten vollzogenen Tertiärisierung der Innenstadtlagen als Ergebnis eines standörtlichen Verdrängungswettbewerbs existiert dort nach wie vor in erheblichem Umfang Wohnraum.

\footnotetext{
${ }^{3}$ Für eine genaue Beschreibung vgl. http://help.arcgis.com/en/arcgisdesktop/10.0/help/index.html\#//00310000002t000000 (20.01.2014).

${ }^{4}$ Unter Dienstleistungsbereich sind folgende Wirtschaftsabschnitte gemäß der Klassifikation der Wirtschaftszweige (WZ) 2008 zu verstehen: J (Informationen und Kommunikation), K (Erbringung von Finanz- und Versicherungsdienstleistungen), L (Grundstücks- und Wohnungswesen), M (Erbringung von freiberuflichen, wissenschaftlichen und technischen Dienstleistungen), N (Erbringung von sonstigen wirtschaftlichen Dienstleistungen) und S (Erbringung von sonstigen Dienstleistungen). Auch hier werden lediglich die sozialversicherungspflichtig Beschäftigten (SVB) berücksichtigt, zusätzlich die geringfügig Beschäftigten. Diese gehen pauschal mit 20\% der Arbeitszeit eines Vollbeschäftigten in Abb. 6 ein, das heißt, die Werte repräsentieren Vollzeit-SVB plus $0,2 *$ geringfügig SVB.
} 


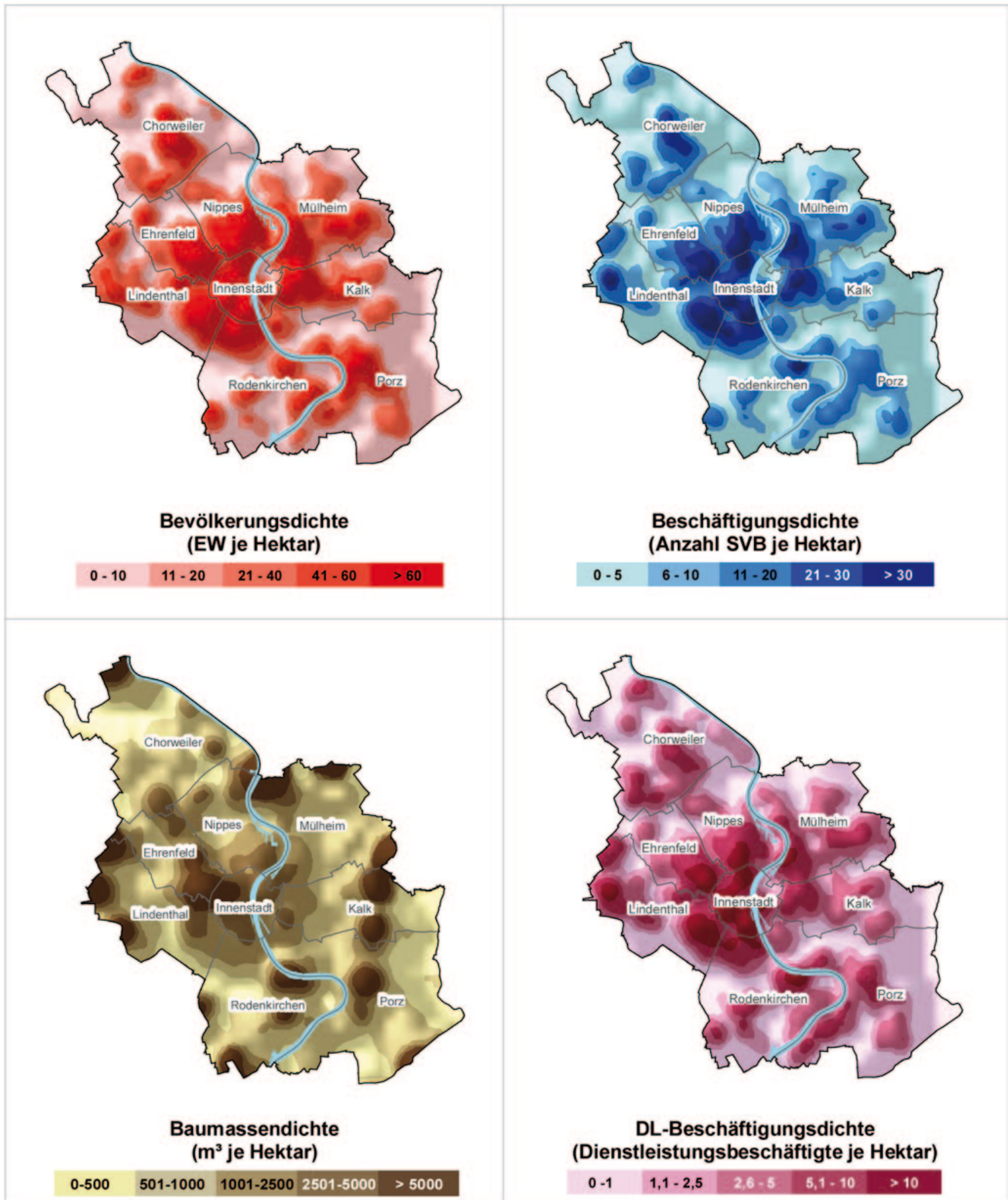

Abb. 6 Morphologische Dichtekarten der Stadt Köln

Darüber hinaus sind in den äußeren Stadtbezirken Subzentren hoher Wohndichte erkennbar, z. B. in Chorweiler, im östlichen Mülheim oder im Süden entlang der Rhein- schleife im Grenzbereich Rodenkirchen/Porz. Auffällig ist die hohe Übereinstimmung der räumlichen Ausprägung von Einwohner- und Arbeitsplatzdichte, auch der Dienst- 
leistungs-Arbeitsplatzdichte. Daraus lassen sich erste Hinweise auf eine grundsätzlich gegebene Nutzungsmischung ableiten: Die räumliche Nähe von Einwohnerkonzentrationen und Arbeitsmöglichkeiten scheint gegeben. Bei den Baumassen dagegen zeigt sich ein leicht anderes Bild: Hier sind im Stadtzentrum die höchsten Werte konzentriert, was sich vor allem auf den hohen Anteil von öffentlichen Bauten, die keine oder nur teilweise Wohn- oder Beschäftigungsfunktion haben, zurückführen lässt. Die höchsten Einwohnerdichten hingegen reichen weiter in die unmittelbar anschließenden Stadtteile.

\subsection{Analyse von städtischen Zentrenstrukturen}

Kleinräumige Dichtedaten können auch für eine Untersuchung der kernstädtischen Zentrenstrukturen verwendet werden. Im Allgemeinen hat in den meisten deutschen Großstädten die Dominanz der ursprünglichen Zentren - vor allem der historischen Innenstadt - im Arbeitsplatzangebot für den gesamten städtischen Raum seit den 1950er Jahren abgenommen. Die heutige Zentrenstruktur von Stadtregionen hat sich stattdessen im Lauf der Jahrzehnte zu einem komplexen, funktional arbeitsteilig organisierten Netzwerk von Zentren und Subzentren entwickelt (vgl. Thierstein/ Lüthi/Kruse et al. 2008; Hesse 2010; Knapp/Volgmann 2011). Wie aber solche metropolitanen Zentrensysteme strukturell beschaffen sind, in welchem Verhältnis sich beispielsweise die Arbeitsplätze auf klassische Innenstadtlagen und randstädtische Standorte verteilen, ist bislang empirisch kaum fassbar.

Eine hier angewendete Methode erfolgt durch eine Analyse der räumlichen Autokorrelation der Dichtewerte mithilfe des Anselin Local Moran's I (vgl. Anselin 1995). Dabei wurden solche Standorte (Baublöcke) als potenzielle Zentren betrachtet, die sich in ihrer Dichteausprägung in statistisch signifikantem Maße von benachbarten Baublöcken unterscheiden (,Spots“) bzw. ihnen ähneln (Clusterbildung). Im Gegensatz zur kartographischen Klassenbildung, die lediglich einen visuellen Eindruck über die Dichteverteilung liefert, erfolgt hier eine auf einer Umgebungsanalyse basierenden Abgrenzung von Zentren. ${ }^{5}$ Mithilfe iterativer Zufallsverteilungen der vorkommenden Werte werden die Abweichungen der tatsächlichen Werteverteilung von der Zufallsverteilung gemessen und als Index-Wert inklusive $z$-Score und $p$-Value für jeden Datenpunkt ausgegeben. Als signifikant werden solche Werte bezeichnet, deren $p$-Value gemäß der Anwendung der False Discovery Rate (FDR)

\footnotetext{
${ }^{5}$ Nachbarschaft wird hier als „queen contiguity of second order" modelliert, das heißt, auch Nachbarn, die sich nur in einem Punkt berühren, sowie die ersten Nachbarn der Nachbarn werden berücksichtigt.
}

kleiner als der jeweils zugehörige kritische Wert ist (Caldas de Castro/Singer 2006: 187). ${ }^{6}$

Im Ergebnis stehen vier Klassen räumlicher Autokorrelation: Blöcke hoher Dichte umgeben von Blöcken hoher Dichte $(\mathrm{HH})$, Blöcke hoher Dichte umgeben von Blöcken niedriger Dichte (HL), Blöcke niedriger Dichte umgeben von Blöcken hoher Dichte (LH) sowie Blöcke niedriger Dichte umgeben von Blöcken niedriger Dichte (LL). Von besonderem Interesse sind die ersten beiden Kategorien, da sie typisch für eine Zentrenausbildung sind (vgl. z. B. Riguelle/Thomas/Verhetsel 2007). Mit Hilfe von LISA Cluster Maps ${ }^{7}$ können statistisch signifikante (Sub-)Zentren der Einwohner-, Arbeitsplatz- und Baumassendichte abgegrenzt und damit räumlich-funktionale Strukturmuster in ein Monitoring eingestellt werden. Bei dem Vergleich der kartographischen Ergebnisse und ihrer Interpretation muss berücksichtigt werden, dass statistische Signifikanz nicht zwangsläufig eine absolut betrachtete hohe Dichte bedeutet. Vielmehr gibt die Signifikanz an, wie die Dichte eines betrachteten Baublocks im Verhältnis zu seinen Nachbarn ausgeprägt ist. Ist der Wert für den Baublock in diesem Kontext ,herausragend" (das heißt, der p-Wert des LISAIndexes ist kleiner als der kritische p-Wert gemäß der False Discovery Rate), kann von Signifikanz gesprochen werden.

Der Vergleich zwischen der Arbeitsplatzdichte aller sozialversicherungspflichtig Beschäftigter und der Arbeitsplatzdichte im Dienstleistungsbereich (vgl. rechte Karten in Abb. 6) zeigt eine hohe Übereinstimmung: Bereiche, in denen die Beschäftigung sehr hoch ist, haben auch eine hohe Beschäftigung im Dienstleistungsbereich. Die erzeugten LISA Cluster Maps (vgl. Abb. 7) erlauben hingegen weitergehende Bewertungen der Zentrenstrukturen. Die roten Punkte in der LISA Cluster Map repräsentieren die Flächengrößen zusammenhängender ATKIS-Flächen hoher Dichte, die von anderen Flächen hoher Dichte umgeben sind (HHCluster). Die blauen Punkte repräsentieren in ihrer Größe Flächen hoher Dichte, die von Flächen relativ niedriger Dichte umgeben sind (HL, „Hot Spots“).

Betrachtet man zunächst die Einwohnerdichte (vgl. Abb. 7, oben links), so lassen sich die Wohnschwerpunkte der Stadt räumlich deutlich herausstellen. Es bestätigt sich das bereits aus der Dichtekarte abgeleitete Ergebnis, wonach in einem Ring um die Innenstadt und punktuell auch in den Verdichtungskernen der Randlagen die höchsten Einwohnerdichten zu finden sind. Darüber hinaus wird

\footnotetext{
${ }^{6}$ Die False Discovery Rate wurde gewählt, da zum einen eine positive räumliche Autokorrelation in den Daten vorliegt. Nach Anselin (1995: 95 f.) sind zum anderen die ,klassische' Rate von 5\% zu wenig restriktiv und eine Family Wise Error Rate nach der von ihm zitierten Bonferroni und Šidák-Methode zu konservativ. Die False Discovery Rate ist dabei ein guter Kompromiss und zudem auch unter positiver räumlicher Abhängigkeit gültig (Benjamini/Yekutieli 2001: 1168)

${ }^{7}$ LISA steht für „Local Indicators of Spatial Autocorrelation“.
} 


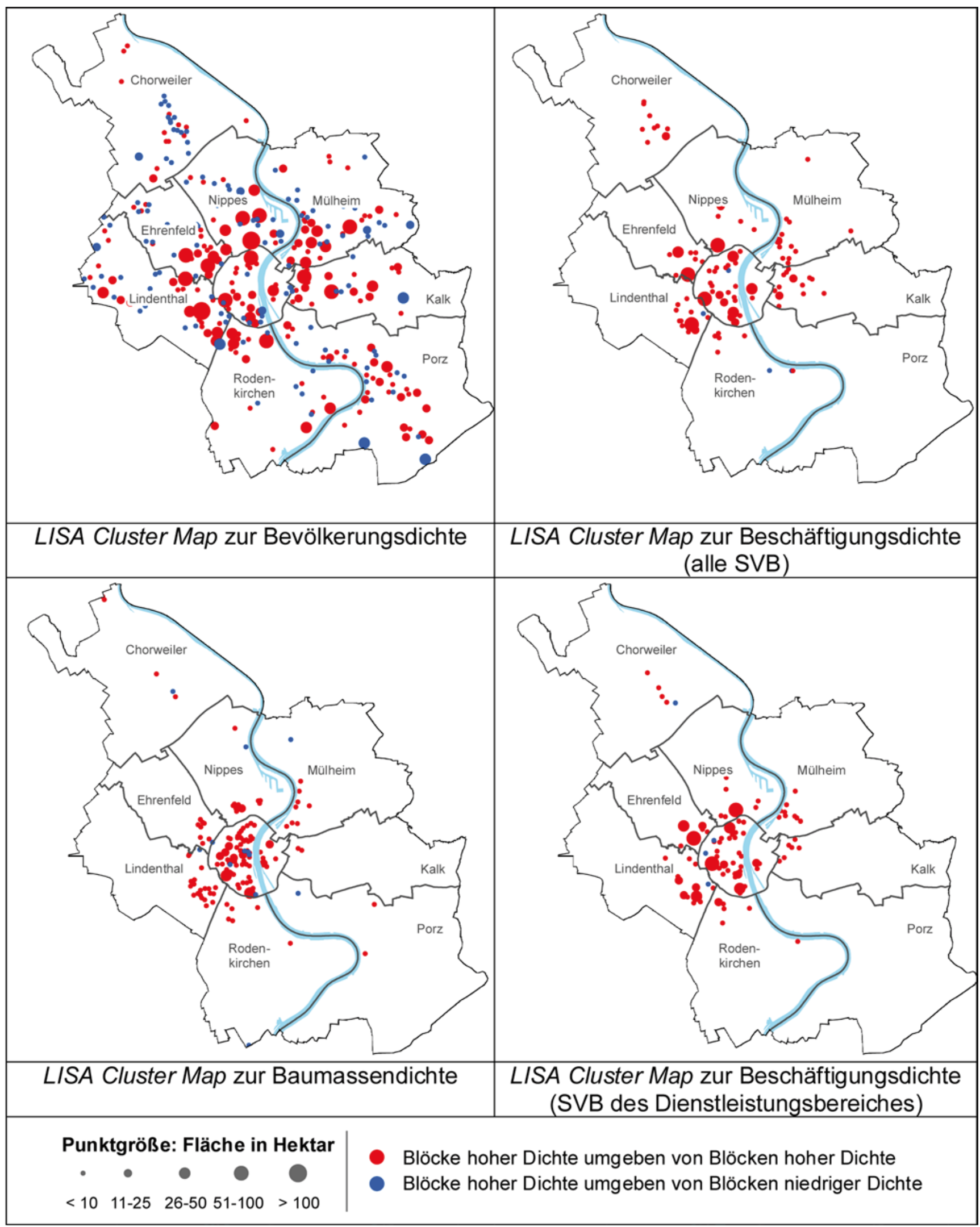

Abb. 7 Räumliche Korrelation von Dichtekennwerten in der Stadt Köln 
für die blauen Konzentrationen aufgezeigt, wo Wohnschwerpunkte isoliert liegen und von anderen Nutzungen umgeben sind. Dies ist außerhalb der Innenstadt vor allem in Chorweiler der Fall, in geringen Ausprägungen auch verstreut in den Randlagen der Stadtregion. Im Bereich der Arbeitsplatzdichte zeigt sich eine deutliche Konzentration von Schwerpunkten im Zentrum und im zentrumsnahen Innenstadtbereich. Diese sind in der Mehrzahl als Cluster hoher Dichte in rot erkennbar, gleichermaßen für Gesamt(oben rechts) und Dienstleistungsbeschäftigung (unten rechts). Großflächige isolierte Beschäftigungszentren sind nicht erkennbar. Anders stellt sich dies bei der räumlichen Verteilung der Baumassendichten (unten links) dar. Hier sind natürlich ebenfalls im und um das Zentrum statistisch signifikante Cluster ähnlich (hoher) Baumassendichten zu finden. Mit zunehmender Entfernung zum Zentrum werden diese jedoch spärlicher und bilden nur noch in den Kernbereichen der Subzentren Cluster aus. Bemerkenswert ist, dass es hier großflächige isolierte Baublockcluster in blau gibt, die sich deutlich von ihrer Umgebung abgrenzen, z. B. in der größten Ausprägung im Stadtteil Nippes die Hafenanlagen in Niehl und die Fordwerke, aber auch im Westen von Rodenkirchen die Industriegebiete südlich von Hürth.

\subsection{Ausschöpfung der planungsrechtlich möglichen Dichte}

Abschließend wird eine weitere Anwendungsoption kleinräumiger Dichtedaten aufgezeigt. Sie stellt eine Analyse der realen Ausschöpfung der planungsrechtlich möglichen Dichte dar. Aus früheren Studien ist bekannt, dass die realen Baudichten die planungsrechtlich mögliche Verdichtung zum Teil drastisch unterschreiten (Losch 1994: 136). Eine Ausnahme kann für Zentrumslagen angenommen werden, wo punktuell deutliche Überschreitungen als Ergebnis von Befreiungen von den baurechtlichen Vorschriften zu erwarten sind. Genauere Erkenntnisse, in welchem Umfang die realen Dichten die in der Baunutzungsverordnung vorgegebenen Obergrenzen unterschreiten (oder auch punktuell überschreiten), gibt es aber bislang nicht. Der hier vorgenommene Abgleich von Plan- und Solldichten erfolgt allein unter Bezugnahme auf den Flächennutzungsplan der Stadt Köln und die darin erfolgte Darstellung von Nutzungsarten. Bebauungspläne und die in ihnen festgesetzten Dichten konnten im Rahmen dieses Beitrags nicht einbezogen werden, weshalb die Ergebnisse nur einen generalisierten Überblick über die Ausnutzung der möglichen Dichte geben.

Der digitale Flächennutzungsplan der Stadt Köln enthält 2.113 Flächen mit den in Tab. 2 dargestellten Nutzungstypen und ihren Flächen. ${ }^{8}$ Für diese Nutzungstypen wurden aus der Baunutzungsverordnung (BauNVO) die

\footnotetext{
${ }^{8}$ Teilweise zusammengefasst bei gleichen Nutzungstypen mit unterschiedlichen Schreibweisen.
}

Tab. 2 Flächennutzungstypen Stadt Köln mit gesetzlichen Grundflächen- (GRZ) und Geschossflächenzahlen (GFZ)

\begin{tabular}{lrll}
\hline Nutzungstyp & Fläche (ha) & GRZ & GFZ \\
\hline Bahn & 13 & - & - \\
Besonderes Wohngebiet & 822 & 0,6 & 1,6 \\
Fläche für die Forstwirtschaft & 2.846 & - & - \\
(Erholungswald) & & & \\
Fläche für die Landwirtschaft & 2.891 & - & - \\
Fläche für die Ver- und Entsorgung & 229 & - & - \\
Fläche für Windenergieanlagen & 21 & - & - \\
Gemeinbedarfsfläche & 527 & - & - \\
Gewerbefläche & 1.968 & 0,8 & 2,4 \\
Grünfläche & 544 & - & - \\
Grünfläche mit besonderer Nutzung & 23 & - & - \\
Grünfläche mit teilweise landwirt- & 11.112 & - & - \\
schaftlicher Nutzung & & & \\
Industriefläche & 1.957 & 0,8 & 2,4 \\
Kerngebiet & 96 & 1,0 & 3,0 \\
Mischbaufläche & 783 & 0,6 & 1,2 \\
Sonderbaufläche & 1.043 & 0,8 & 2,4 \\
Verkehrsfläche & 37 & - & - \\
Wohnbaufläche & 8.446 & 0,4 & 1,2 \\
\hline
\end{tabular}

Geschossflächenzahl (GFZ) und die Grundflächenzahl (GRZ) recherchiert. Ein Abgleich dieser theoretisch realisierbaren Geschossflächen erfolgte über die Auswertung der oben beschriebenen fernerkundlich erhobenen Baumassen (vgl. Kap. 3). Zunächst wurde für die ATKIS-Flächen die dominante Flächennutzung ermittelt. Das oben beschriebene Umlegungsverfahren ermöglicht im Weiteren eine Zuordnung der Gebäude bzw. Gebäudeteile zu den Grundflächen der baulich geprägten ATKIS-Flächen einschließlich der Angabe der Anzahl der Stockwerke. Die geschätzte, tatsächlich verbaute Geschossflächenzahl ergibt sich letztlich aus den auf Blockebene aggregierten Geschossflächen im Verhältnis zur Grundfläche des Baublocks. Dabei ist zu beachten, dass sich Verzerrungen gegenüber einer städtebaulich korrekt berechneten Geschossflächenzahl aus einer abweichenden Grundfläche der ATKIS-Flächen gegenüber den Grundflächen eines Bebauungsplanes ergeben können, für den die Geschossflächenzahl letztlich Rechtsgültigkeit hat. Zwar wird die Reduktion um Verkehrs- und Grünflächen sowohl im Bebauungsplan als auch im Datenmodell von ATKIS vorgenommen, allerdings nicht nach dem gleichen Regelwerk und in einem anderen Detaillierungsgrad.

Der letzte Analyseschritt trägt die Ausnutzung der erlaubten baulichen Dichte durch die geschätzte tatsächliche Dichte im Ringzonenmodell um das Stadtzentrum der Stadt Köln auf. Auf der X-Achse in Abb. 8 sind die Distanzen in Kilometer zum Kölner Dom angegeben, auf der Y-Achse die flächengewichtete mittlere Ausnutzung in Prozent pro Nutzungstyp (in Farbe) und im Mittel aller Flächennutzungstypen (schwarz). Die Ergebnisse verdeutlichen ein starkes Zentrum-Stadtrand-Gefälle in der Ausnutzung der theoretisch möglichen baulichen Dichte, vor allem in Bezug auf 
Abb. 8 Nutzungsintensität verschiedener Flächennutzungstypen nach Distanz vom Stadtzentrum Köln (Nutzungsintensität: planungsrechtlich mögliche GFZ nach BauGB versus GFZ-Schätzung über das DOM)

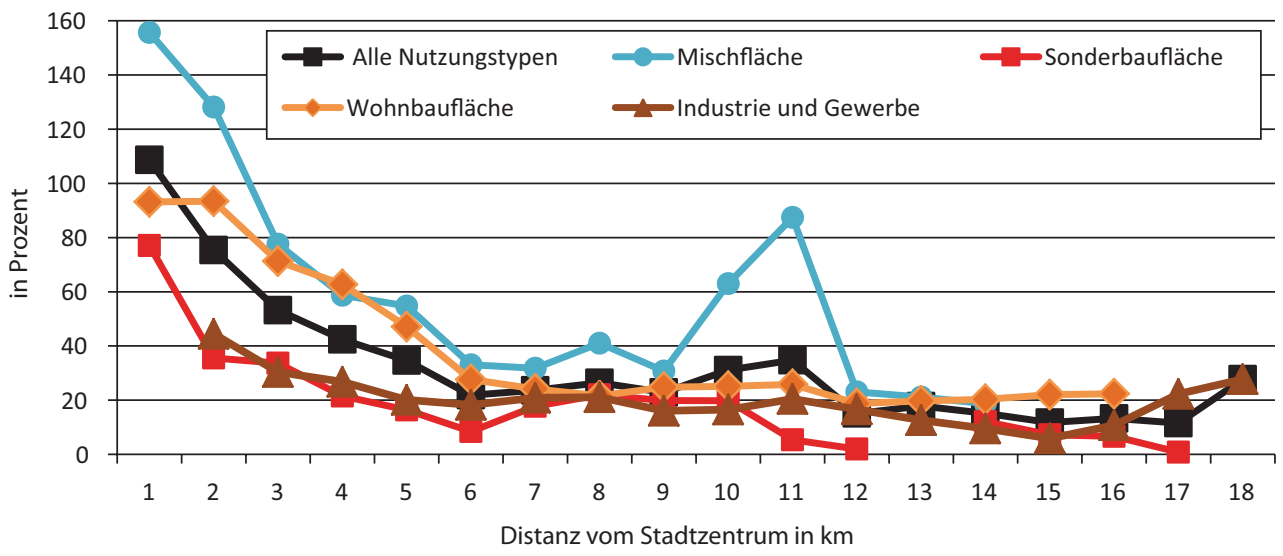

Flächen mit Wohnfunktion sowie Mischflächen. Für Mischflächen ist aus historischen Gründen im Stadtzentrum eine Übernutzung der nach Baunutzungsverordnung zulässigen Geschossflächenzahl zu erkennen. Es wird aber auch deutlich, dass außerhalb des innerstädtischen Zentrums (etwa ab $2 \mathrm{~km}$ ) die Nutzungseffizienz stark abnimmt und eine generelle Unterschreitung der möglichen Dichtegrenzwerte zu verzeichnen ist. Des Weiteren zeigt sich bei Kilometer 11 eine Spitze (peak) für Mischflächen ( $80 \%$ der erlaubten Geschossflächenzahl werden genutzt). Verantwortlich hierfür sind Flächen im Zentrum der Großwohnsiedlung Chorweiler mit GFZ-Überschreitungen in einzelnen Baublöcken von über $300 \%$.

Bei der Interpretation dieser Ergebnisse ist zu berücksichtigen, dass eine hohe Nutzungseffizienz nicht zwangsläufig wünschenswert ist. Neben ökologischen Aspekten wie der Stadtklimatologie oder dem Hochwasserschutz sind selbstverständlich auch die Freiraumversorgung und Durchgrünung der Stadt sowie die städtebauliche Qualität und der Bebauungscharakter von Wohnquartieren zu berücksichtigen, die häufig im Konflikt mit einer höheren Ausnutzung der zulässigen Geschossflächenzahl stehen. Aus dieser Perspektive liegt der Wert der vorliegenden Analyse in einer Beschreibung des Ist-Zustandes. Um einen konkreten Mehrwert für die Stadtplanung zu erzeugen, sind weiterführende Analysen zu Nachverdichtungspotenzialen unerlässlich. Die vorliegenden Datengrundlagen können hier lediglich informatorisch unterstützen.

\section{Fazit und Ausblick}

Der vorliegende Beitrag zeigt, dass mit Hilfe von Fernerkundungsdaten die Bereitstellung gebäudescharfer 3D-Informationsgrundlagen aus technischer Sicht qualitativ hochwertig möglich ist. Die dabei erzeugten Daten zu Baumassen und Bauvolumen schließen eine Lücke für das Monitoring der Stadtentwicklung, denn insbesondere die stadtregional vergleichbare Berechnung von Geschossflä- chen- und Bebauungsdichten war bislang nur mit bedeutend höherem Aufwand realisierbar. Für eine flächendeckende Umsetzung der beschriebenen Methoden sind der Kostenaufwand für die Akquise und die Prozessierung der Erdbeobachtungsdaten derzeit zwar nach wie vor relativ hoch. Es kann aber mit zukünftigen Effizienzsteigerungen gerechnet werden, die schon heute gewisse Investitionen in die Methodenentwicklung rechtfertigen.

Diesbezüglich muss festgehalten werden, dass die Vernetzung von 3D-Stadtmodellen, Geobasisdaten und räumlicher Statistik methodisch noch in den Anfängen steckt. Die Harmonisierung von Datenformaten und der organisatorische Datenaustausch stellen den Anwender vor große Herausforderungen, und man ist hier von der Entwicklung wünschenswerter standardisierter Prozesse noch weit entfernt. Gleichzeitig verdeutlichen die dargestellten thematischen Anwendungen aber den universellen Nutzen derartig kombinierter Datenquellen in der Form kleinräumiger Dichtedaten.

Sowohl aus planungspraktischer als auch aus raumwissenschaftlicher Perspektive eröffnen sich damit vielfältige Nutzungsoptionen. Beispielhafte Anwendungsbezüge für das Monitoring der Dichte bieten sich in

- der Stadtklimatologie und hier insbesondere bei der methodischen Weiterentwicklung von Vulnerabilitätsstudien (z. B. Berechnung von Verschattungsverhältnissen),

- der Planung des Stadtumbaus, indem unter anderem Rückbauoptionen auf ihre infrastrukturellen Implikationen vertiefend untersucht werden,

- der Bewertung von Nachverdichtungsmaßnahmen mit ihren positiven und negativen Auswirkungen (z. B. Verbesserung der Infrastruktureffizienz, Verringerung der Freiraumversorgung) oder

- der Durchführung von Energieverbrauchs- und -einsparszenarien im Gebäudebereich, für die belastbare Daten zu Gebäudemassen und -formen von großer Bedeutung sind. 
Als grundsätzlich sinnvoll wird die Ergänzung konventioneller Monitoringsysteme auf kommunaler und regionaler Ebene eingeschätzt, indem Dichteindikatoren integriert und mit anderen Indikatoren kombiniert werden können. Der Vorteil der hier aufgezeigten Methoden liegt vor allem darin, dass ein multidimensionales Dichtemonitoring für größere Gebietskulissen bei regelmäßiger Fortschreibung der Daten mit vertretbarem Aufwand leistbar ist. Die damit erleichterte Verfügbarkeit teilgemeindlicher Daten ermöglicht auch interregional vergleichende Analysen siedlungsstruktureller Zustände und Entwicklungen, die für Deutschland so bisher nicht möglich waren. Auch im Hinblick auf erwartbare Steigerungen der Kosteneffizienz der fernerkundlichen Verfahren kann eine Weiterentwicklung und Adaption der hier vorgestellten Methoden für die Stadtforschung und angrenzende Disziplinen deshalb nur empfohlen werden.

Danksagung Die Autoren bedanken sich bei der Deutschen Forschungsgemeinschaft (DFG) für die Finanzierung des Forschungsprojektes „Polyzentralität deutscher Stadtregionen“ mit dem Förderkennzeichen: TA 800/1-1 \& SI 932/4-1. Darüber hinaus bedanken sich die Autoren bei Udo Lapschies vom Umwelt- und Verbraucherschutzamt der Stadt Köln und bei Ute Meurer der Bezirksregierung Köln für die Bereitstellung der Geodaten. Unser Dank gilt schließlich auch den Mitarbeitern im Amt für Stadtentwicklung und Statistik der Stadt Köln, die uns mit der Bereitstellung von kleinräumigen Bevölkerungsdaten sehr unterstützt haben sowie dem Forschungsdatenzentrum der Bundesagentur für Arbeit im Institut für Arbeitsmarkt- und Berufsforschung (IAB) für die Aggregation der Beschäftigtendaten.

\section{Literatur}

Acioly Jr., C.; Davidson, F. (1996): Density in urban development. In: Building Issues 8 (3), 3-25.

Ackermann, F. (1999): Airborne laser scanning - present status and future expectations. In: Journal of Photogrammetry and Remote Sensing 54 (2-3), 64-67.

Albertz, J. (2001): Einführung in die Fernerkundung. Grundlagen der Interpretation von Luft- und Satellitenbildern. Darmstadt.

Anselin, L. (1995): Local Indicators of Spatial Association - LISA. In: Geographical Analysis 27 (2), 93-115.

Benjamini, Y.; Yekutieli, D. (2001): The control of the false discovery rate in multiple testing under dependency. In: The Annals of Statistics 29 (4), 1165-1188.

Bettencourt, L.; West, G. (2010): A unified theory of urban living. In: Nature 467, 912-913.

Bundesagentur für Arbeit (2012): Statistische Geheimhaltung: Rechtliche Grundlagen und fachliche Regelungen der Statistik der Bundesagentur für Arbeit. Nürnberg.

Burchell, R. W.; Mukherji, S. (2003): Conventional development versus managed growth: the costs of sprawl. In: American Journal of Public Health 93 (9), 1534-1540.

Caldas de Castro, M.; Singer, B. H. (2006): Controlling the False Discovery Rate: A New Application to Account for Multiple and Dependent Tests in Local Statistics of Spatial Association. In: Geographical Analysis 38 (2), 180-208.

Churchman, A. (1999): Disentangling the concept of density. In: Journal of Planning Literature 13 (4), 389-411.
Doubek, C.; Zanetti, G. (1999): Siedlungsstruktur und öffentliche Haushalte. Wien. $=$ Schriftenreihe der Österreichischen Raumordnungskonferenz 143.

Ewing, R.; Cervero, R. (2010): Travel and the built environment. A meta-analysis. In: Journal of the American Planning Association 76 (3), 265-294.

Ewing, R.; Bartholomew, K.; Winkelman, S.; Walters, J.; Chen, D. (2007): Growing cooler. The evidence on urban development and climate change. Washington, DC.

Forsyth, A. (2003): Measuring density: working definitions for residential density and building intensity. Minneapolis. = Design Brief, Number 8, July 2003. http://www.corridordevelopment.org/ pdfs/from_MDC_Website/db9.pdf (17.01.2014).

Forsyth, A.; Oates, J. M.; Schmitz, K. H.; Hearst, M. (2007): Does residential density increase walking and other physical activity? In: Urban Studies 44 (4), 679-697.

Frank, L. D.; Engelke, P. O. (2001): The built environment and human activity patterns: Exploring the impacts of urban form on public health. In: Journal of Planning Literature 16 (2), 202-218.

Gassner, E. (1994): Die Grenzen der Verdichtung bei Wohnbaugebieten. In: Borchard, K., Weiß, E. (Hrsg.): Aus Verantwortung für die Gestaltung unserer Umwelt. Ausgewählte Beiträge von Edmund Gassner. Bonn, 93-276. = Beiträge zu Städtebau und Bodenordnung 12.

Hesse, M. (2010): Reurbanisierung oder Metropolisierung? Entwicklungspfade, Kontexte, Interpretationsmuster zum aktuellen Wandel der Großstadtregionen. In: DISP 46 (180), 36-46.

Hinde, S.; Dixon, J. (2005): Changing the obesogenic environment: insights from a cultural economy of car reliance. In: Transportation Research Part D 10 (1), 31-53.

Kelly-Schwartz, A. C.; Stockard, J.; Doyle, S.; Schlossberg, M. (2004): Is sprawl unhealthy? A multilevel analysis of the relationship of metropolitan sprawl to the health of individuals. In: Journal of Planning Education and Research 24 (2), 184-196.

Knapp, W.; Volgmann, K. (2011): Neue ökonomische Kerne in nordrhein-westfälischen Stadtregionen: Postsuburbanisierung und Restrukturierung kernstädtischer Räume. In: Raumforschung und Raumordnung 69 (5), 303-317.

Koppe, C.; Kovats, S.; Jendritzky, G.; Menne, B. (2004): Heat-waves: risks and responses. Kopenhagen. $=$ Health and Global Environmental Change Series 2.

Kropp, J.; Holsten, A.; Lissner, T.; Roithmeier, O.; Hattermann, F.; Huang, S.; Rock, J.; Wechsung, F.; Lüttger, A.; Pompe, S.; Kühn, I.; Costa, L.; Steinhäuser, M.; Walther, C.; Klaus, M.; Ritchie, S.; Metzger, M. (2009): Klimawandel in Nordrhein-Westfalen. Regionale Abschätzung der Anfälligkeit ausgewählter Sektoren. Abschlussbericht des Potsdam-Instituts für Klimafolgenforschung (PIK) für das Ministerium für Umwelt und Naturschutz, Landwirtschaft und Verbraucherschutz Nordrhein-Westfalen (MUNLV). Potsdam.

Landeshauptstadt Stuttgart (2005): Stadträumliche Dichte. Flächenbilanz von Wohngebieten in Stuttgart. Stuttgart.

Lillesand, T. M.; Kiefer, R. W.; Chipman, J. W. (2004): Remote Sensing and image interpretation. Hoboken, New Jersey.

Losch, S. (1994): Baulandreserven im Bestand. In: BfLR - Bundesforschungsanstalt für Landeskunde und Raumordnung (Hrsg.): Bestand, Bedarf und Verfügbarkeit von Baulandreserven. Umfrageergebnisse und Regionalerhebungen. Bonn, 135-142. = Materialien zur Raumentwicklung 64.

Newman, P.; Hogan, T. (1981): A review of urban density models: toward a resolution of the conflict between populace and planner. In: Human Ecology 9 (3), 269-303.

Newman, P.; Kenworthy, J. R. (1989): Gasoline Consumption and Cities. A Comparison of U.S. Cities with a Global Survey. In: Journal of the American Planning Association 55 (1), 24-37.

OECD (2010): Cities and Climate Change. Paris. 
OECD (2012): Compact City Policies. A Comparative Assessment. Paris.

Riguelle, F.; Thomas, I.; Verhetsel, A. (2007): Measuring urban polycentrism: a European case study and its implications. In: Journal of Economic Geography 7 (2), 193-215.

Roberts, B. H. (2007): Changes in urban density: Its implications on the sustainable development of Australian cities. http://soac.fbe.unsw. edu.au/2007/SOAC/changesinurbandensity.pdf (20.01.2014).

Roskamm, N. (2011): Dichte. Eine transdisziplinäre Dekonstruktion. Bielefeld.

Scholz, T.; Rauscher, C.; Reiher, J.; Bachteler, T. (2012): Geocoding of German Administrative Data: The Case of the Institute for Employment Research. http://doku.iab.de/fdz/reporte/2012/MR_0912_EN.pdf (17.01.2014).

Senatsverwaltung für Stadtentwicklung und Umwelt Berlin (2013): Umweltatlas Berlin, Themenbereich Einwohnerdichte. Berlin. http:/www.stadtentwicklung.berlin.de/umwelt/umweltatlas/ii606. htm (17.01.2014).

Siedentop, S.; Schiller, G.; Gutsche, J.-M.; Koziol, M.; Walther, J. (2006): Siedlungsentwicklung und Infrastrukturfolgekosten. Bilanzierung und Strategieentwicklung. Bonn.= BBR-Online-Publikationen 3/2006.
Thierstein, A.; Lüthi, S.; Kruse, C.; Gabi, S.; Glanzmann, L. (2008): Changing value chain of the Swiss knowledge economy: spatial impact of intra-firm and inter-firm networks within the emerging mega-city region of Northern Switzerland. In: Regional Studies 42 (8), 1113-1131.

Watkins, R.; Palmer, J.; Kolokotroni, M. (2007): Increased temperature and intensification of the urban heat island: Implications for human comfort and urban design. In: Built Environment 33 (1), $85-96$.

Westphal, C. (2008): Dichte und Schrumpfung. Kriterien zur Bestimmung angemessener Dichten in Wohnquartieren schrumpfender Städte aus Sicht der stadttechnischen Infrastruktur. Dresden. = IÖR-Schriften 49.

Wurm, M. (2013): Verknüpfung von Fernerkundungsdaten und Survey-Daten (SOEP und BASE-II) in städtischen Räumen für sozialwissenschaftliche Analysen. Dissertation an der Technischen Universität Graz.

Wurm, M.; Taubenböck, H.; Schardt, M.; Esch, T.; Dech, S. (2011): Object-based image information fusion using multisensor earth observation data over urban areas. In: International Journal of Image and Data Fusion 2 (2), 121-147. 